\title{
8 Literatur und Adressen
}

\subsection{Literatur}

[1] Benedikt MA, Weitgasser R. Ernährungsmanagement bei Wundpatienten. J Ernährungsmed 2006; 8(2): 6-10

[2] Deutsches Netzwerk für Qualitätsentwicklung in der Pflege (Hrsg.). Expertenstandard „Pflege von Menschen mit chronischen Wunden“. 1. Aktualisierung 2015. Im Internet: www.dnqp.de/fileadmin/HSOS/Homepages/DNQP/Dateien/Expertenstandards/Pflege_von_Menschen_mit_chronischen_Wunden/ChronWu_Akt_Auszug.pdf; Stand: 21.07.2021

[3] Horn T et al. Krefelder Wundfibel 2005

[4] Kondrup J et al. Clinical Nutrition 2003; 22: 415-421. Empfohlen von der Europäischen Gesellschaft für Klinische Ernährung und Stoffwechsel (ESPEN).

[5] Sailer M. Patientenedukation. In: Panfil EM, Schröder G. Pflege von Menschen mit chronischen Wunden. 1. Auflage. Bern: Hans Huber; 2010: 453

[6] Schmidt S. Expertenstandard Ernährungsmanagement zur Sicherstellung und Förderung der oralen Ernährung in der Pflege. In: Expertenstandards in der Pflege - eine Gebrauchsanleitung. 4. Aufl. Berlin, Heidelberg: Springer; 2020: 147-169

[7] Schreier MM, Bartholomeyczik S. Die Rolle der Pflege bei der Ernährung im Krankenhaus. Aktuel Ernaehr Med 2008; 33: 70-74

\subsection{Nationale Adressen}

\author{
Arbeitsgemeinschaft der Wissenschaftlichen Medizinischen \\ Fachgesellschaften e. V. (AWMF) \\ Birkenstr. 67 \\ D-10 559 Berlin \\ Tel.: + 493020097777 \\ Fax: nur auf Anfrage \\ E-Mail: office@awmf.org \\ Web: www.awmf.org
}

\section{Arbeitsgemeinschaft „Fuß“ der Deutschen Diabetologischen Gesellschaft Bettina Baumann}

Postfach 28

D-63 774 Mömbris

E-Mail: info@ag-fuss-ddg.de

Web: www.ag-fuss-ddg.de 
Deutsche Gesellschaft für Wundheilung und Wundbehandlung e.V.

Geschäftsstelle

Glaubrechtstraße 7

D-35392 Gießen

E-Mail: dgfw@dgfw.de

Web: www.dgfw.de

Initiative Chronische Wunden e. V. (ICW )

Wipertistr. 1a (Wipertihof)

D-06 484 Quedlinburg

E-Mail: organisation@icwunden.de

Web: www.icwunden.de

Robert-Koch-Institut

Nordufer 20

13353 Berlin

Tel.: + 4930187540

Fax: + 4930187542328

E-Mail: webmaster@rki.de

Web: www.rki.de

\subsection{Internationale Adressen}

Deutsch-Österreichisch-Schweizerische Wundheilungsorganisation (Wund-D.A.CH)

Gyrowetzgasse 8

A-1140 Wien

Tel.: + $43(0) 16022548$

Fax: + 43 (0)1 60225 48-90

E-Mail: office@wund-dach.org

Web: www.wund-dach.org

European Wound Management Association (EWMA)

Nordre Fasanvej 113, 2.

DK-2000 Frederiksberg

Tel.: + 4570200305

Fax: + 4570200315

E-Mail: ewma@ewma.org

Web: www.ewma.org 
American Professional Wound Care Association (APWCA)

6737 W. Washington St. | Suite 4210

Milwaukee, WI 53 214, USA

Tel.: + 1414488.3913

Fax: + 14142767704

E-Mail: info@apwca.org

Web: www.apwca.org

Wounds Canada (Canadian Association of Wound Care)

PO Box 35569 York Mills Plaza

North York, ON M2 L 2Y4, CA

Tel.: + 1 416-485-2292

Fax: 888-710-2289

Web: www.woundscanada.ca 